\title{
Precision genetic engineering tools for next-generation plant breeding
}

\author{
Günther Hahne ${ }^{1}$ Laurence Tomlinson ${ }^{1}$. Fabien Nogué ${ }^{1}$
}

Received: 26 February 2019 / Accepted: 5 March 2019 / Published online: 28 March 2019

c) Springer-Verlag GmbH Germany, part of Springer Nature 2019

Genetic improvement of plants has come of age. After thousands of years of selecting convenient but fortuitous mutations, and one and a half centuries of increasingly applying the fruits of scientific insight to plant breeding, progress in this domain has reached the molecular level several decades ago. It is probably now, for the first time, that the term "precision" can be used to characterize the technology available for genetic engineering aiming at new agronomic traits in plants. All available evidence indicates that we are indeed on the threshold of a new generation of tools available for plant improvement.

Thus, it appears timely to dedicate an entire special issue to this topic. The eleven contributions in this special issue will shed light on the subject from different angles, and in different formats. Without claiming to be exhaustive, the two opinion papers, one review article, three focus papers and five original papers all contribute to capturing the effectiveness, the limits and the new challenges, of the new genetic engineering tools for different aspects of plant breeding.

Huang and Puchta (2019) consider the available tools for gene editing through homologous recombination, pointing out the current limits and bottlenecks but also providing suggestions on how to use the technology efficiently. Chaudhary (2018) considers the CRISPR/Cas13a System and its potential as an RNA-guided RNA nuclease platform which may complement, or eventually replace, traditional approaches for combating plant RNA viruses. While the CRISPR/Cas system is in the process of becoming one of the standard technologies for genetic engineering, thanks to the precision with which the intended gene sequences can be targeted, one of the most important considerations for practical application is whether we need to worry about off-targeting in

Communicated by Neal Stewart.

Günther Hahne

Weinheim, Germany plants. Hahn and Nekrasov address this question in their opinion paper (Hahn and Nekrasov 2018).

The issue of precise editing of the targeted modifications finds its practical illustration in several of the focus and original articles. The engineering of herbicide resistance, through homologous recombination in tobacco by Hirohata et al. (2018) and through base editing (nCAS9-deaminase) in watermelon, by Tian et al. (2018), show that precise editing is more and more feasible in crops. In addition, Danilo et al. (2019) show that in tomato precise editing can be transgene free, challenging further the frontiers between gene-edited plants and more classical GMOs. In parallel, gene knock-out of multiple genes in maize is reported by Doll et al. (2019) and on a larger scale, Zhou et al. (2018) report on simultaneous introduction of three trait-related QTLs in three rice varieties with multiplex editing by CRISPR-Cas9, resulting in significant yield increase. Introducing mutations with high precision is becoming a well-mastered routine in plant genetic engineering, yet obtaining the desired phenotype may prove to be more complex than that, due to compensatory mechanisms of the plant metabolism. Pérez et al. (2019) illustrate this complexity by showing allele-specific and zygosity-dependent feedback effects on endosperm starch biosynthesis after CRISPR/Cas9-induced mutations.

With multiple gene knock-outs pops up the challenge of efficient identification of the CRISPR/Cas9-induced indels. Biswas et al. (2019) address this question and propose new methods for the selection of indel mutants. Finally, coding genes are not the sole targets of gene knock-out and Saika et al. (2018) report on the structural modification of a locus by targeted deletion of a rice retrotransposon with the final aim of reactivating expression of agronomically important genes.

This special issue addresses some of the most advanced approaches to plant genetic engineering and puts them in perspective with the notion of the degree of precision that one is entitled to expect from their application to real-world problems. Much progress has been made, undoubtedly, but 
the plant genetic and epigenetic machinery is of a complexity that still requires better understanding before the vision of devising a rational plant improvement strategy on the drawing board and translating it faithfully into the desired phenotype with a few precisely targeted interventions, becomes reality. The contributions to this special issue explore this complexity and highlight challenges as much as indicating the way to move forward.

Günther Hahne, Editor-in-Chief

Fabien Nogué, Guest Editor

Laurence Tomlinson, Guest Editor

Acknowledgements We would like to thank the authors, reviewers, and journal editorial staff who have contributed to this special issue.

\section{References}

Biswas S, Li R, Yuan Z et al (2019) Development of methods for effective identification of CRISPR/Cas9-induced indels in rice. Plant Cell Rep. https://doi.org/10.1007/s00299-019-02392-3

Chaudhary K (2018) CRISPR/Cas13a targeting of RNA virus in plants. Plant Cell Rep 37:1707-1712. https://doi.org/10.1007/s0029 9-018-2297-2

Danilo B, Perrot L, Mara K et al (2019) Efficient and transgene-free gene targeting using Agrobacterium-mediated delivery of the
CRISPR/Cas9 system in tomato. Plant Cell Rep. https://doi. org/10.1007/s00299-019-02373-6

Doll NM, Gilles LM, Gérentes MF et al (2019) Single and multiple gene knockouts by CRISPR-Cas9 in maize. Plant Cell Rep. https ://doi.org/10.1007/s00299-019-02378-1

Hahn F, Nekrasov V (2018) CRISPR/Cas precision: do we need to worry about off-targeting in plants? Plant Cell Rep. https://doi. org/10.1007/s00299-018-2355-9

Hirohata A, Sato I, Kaino K et al (2018) CRISPR/Cas9-mediated homologous recombination in tobacco. Plant Cell Rep. https:// doi.org/10.1007/s00299-018-2320-7

Huang TK, Puchta H (2019) CRISPR/Cas-mediated gene targeting in plants: finally a turn for the better for homologous recombination. Plant Cell Rep. https://doi.org/10.1007/s00299-019-02379-0

Pérez L, Soto E, Farré G et al (2019) CRISPR/Cas9 mutations in the rice Waxy/GBSSI gene induce allele-specific and zygositydependent feedback effects on endosperm starch biosynthesis. Plant Cell Rep 38:417-433. https://doi.org/10.1007/s00299-01902388-Z

Saika H, Mori A, Endo M et al (2018) Targeted deletion of rice retrotransposon Tos 17 via CRISPR/Cas9. Plant Cell Rep. https://doi. org/10.1007/s00299-018-2357-7

Tian S, Jiang L, Cui X et al (2018) Engineering herbicide-resistant watermelon variety through CRISPR/Cas9-mediated base-editing. Plant Cell Rep 37:1353-1356. https://doi.org/10.1007/s0029 9-018-2299-0

Zhou J, Xin X, He Y et al (2018) Multiplex QTL editing of grainrelated genes improves yield in elite rice varieties. Plant Cell Rep. https://doi.org/10.1007/s00299-018-2340-3

Publisher's Note Springer Nature remains neutral with regard to jurisdictional claims in published maps and institutional affiliations. 